\title{
Strongly Secure Certificateless Signature Scheme Supporting Batch Verification
}

\author{
Chun-I Fan, ${ }^{1}$ Pei-Hsiu Ho, ${ }^{2}$ and Yi-Feng Tseng ${ }^{1}$ \\ ${ }^{1}$ Department of Computer Science and Engineering, National Sun Yat-sen University, Kaohsiung 80424, Taiwan \\ ${ }^{2}$ Network Benchmarking Lab, Hsinchu 30010, Taiwan \\ Correspondence should be addressed to Chun-I Fan; cifan@faculty.nsysu.edu.tw
}

Received 14 November 2013; Revised 7 March 2014; Accepted 13 March 2014; Published 16 April 2014

Academic Editor: Jian Guo Zhou

Copyright (c) 2014 Chun-I Fan et al. This is an open access article distributed under the Creative Commons Attribution License, which permits unrestricted use, distribution, and reproduction in any medium, provided the original work is properly cited.

\begin{abstract}
We propose a strongly secure certificateless signature scheme supporting batch verification, which makes it possible for a verifier to verify a set of signatures more efficiently than verifying them one by one. In an identity-based digital signature scheme, private key generator (PKG) knows each user's signing key, so it can generate a signature which is indistinguishable from the signature generated by the user. This is a serious problem because the property of signature nonrepudiation will not be achieved. In our proposed scheme, it is impossible for PKG to produce a signature which is indistinguishable from any signature produced by a user. Compared with existing signature schemes with batch verification, although our proposed scheme is not the most efficient one, it achieves Girault's level-3 security, while the others have Girault's level-1 or level-2 security only. We also formally prove that the proposed scheme is unforgeable and satisfies Girault's level-3 security based on hard problems.
\end{abstract}

\section{Introduction}

In traditional certificate-based public-key cryptosystems, a user's public key is produced and is not related to her/his identity. Therefore, the key needs to be certificated by some certification authority (CA) with respect to the user's identity. Anyone who wants to use the public key must verify the validity of the corresponding certificate for the key first. Considering implementation, the management of public key certificates requires a large amount of computation cost and storage.

To reduce the cost of certificate management, Shamir [1] proposed identity based public key cryptography (ID-PKC) in 1984. In ID-PKC, a user's public key can be an arbitrary bit string which can represent the user's identity, such as her/his email address or telephone number. And the user's corresponding private key is computed by a trusted party, called private key generator (PKG) [2].

An inherent problem of ID-PKC is the key escrow problem. That is, the private key of a user is known to PKG. PKG can act as any user to decrypt any ciphertext or generate a signature on any message. To solve this problem,
Al-Riyami and Paterson [3] proposed certificateless public key cryptography (CL-PKC) in 2003. In CL-PKC, a user's secret key is a combination of the secret key, computed by PKG using its master secret key, and a user-chosen secret. Thus, PKG cannot know the complete secret key of the user.

In 2003, a certificateless public-key signature scheme [3] was proposed, but it suffered from the key replacement attack [4]. After that, several certificateless signature schemes [5-18] were introduced recently. In 2007, Hu et al. [19] proposed a new security model and an improved generic construction for certificateless signatures. It showed that certificateless signatures should satisfy the property of Girault's level-3 security [20]. If a certificateless signature scheme meets the above property, the framework of CL-PKC will be with the same security level as that of the traditional certificate-based public key cryptosystems.

In 1989, batch cryptography was first introduced by Fiat [21]. In a signature scheme with batch verification, the cost for verifying $n$ signatures is less than verifying them one by one. After [21], some results [22-24] about batch verification based on RSA or DLP have been proposed. In 2004, Yoon et al. proposed an ID-based signature scheme with batch 
verification [25], but their security proof does not meet the definition of batch verification [26]. After that, some IDbased and group signature schemes with batch verification were proposed in [27-34], respectively, but only [29] is a certificateless signature scheme. Besides, the randomization technique [26, 35-39] for the security of signatures with batch verification was introduced. The technique can withstand the attack that an attacker cheats a verifier to accept invalid signatures.

In this paper, we will design a certificateless signature scheme with efficient batch verification. The computation cost of our scheme in batch verification is only three pairings and it is independent of the number of individual signatures which will be verified. Furthermore, our scheme satisfies Girault's level-3 security. Compared to certificateless, IDbased, and group signature schemes with batch verification $[7,11,14,16,27-34]$, our scheme achieves Girault's level-3 security, while [27-34] meet Girault's level-1 security and [7, $11,14,16,29]$ satisfy Girault's level-2 security only. Compared to $[5,13]$, which also achieve Girault's level-3 security, our scheme is more efficient in verification because of the batch property.

The rest of this paper is organized as follows. In Section 2, we introduce some preliminaries about mathematical backgrounds and definitions. In Section 3, we present the proposed scheme. In Section 4, we provide formal security proofs for our scheme. We compare the proposed scheme with $[5,7,11,13,14,16,27-34]$ in Section 5. Finally, a concluding remark is given in Section 6.

\section{Preliminaries}

In this section, we review the properties of bilinear groups and some related hard problems.

Let $G_{1}$ and $G_{2}$ be two cyclic groups of prime order $q$. Let $P$ be a randomly chosen generator of $G_{1}$ and let $e$ be a bilinear mapping such that $e: G_{1} \times G_{1} \rightarrow G_{2}$, which satisfies the following properties.

(1) Bilinearity: for all $P, Q, R \in G_{1}, e(P+Q, R)=$ $e(P, R) e(Q, R)$ and $e(P, Q+R)=e(P, Q) e(P, R)$.

(2) Nondegeneracy: there exists $P, Q \in G_{1}$ such that $e(P, Q) \neq 1$.

(3) Computability: there exists an efficient algorithm to compute $e(P, Q)$ for any $P, Q \in G_{1}$.

Definition 1 (batch verification of signatures [35]). Let $l$ be the security parameter. Suppose that (Gen,Sign,Verify) is a signature scheme, $n \in \operatorname{polynomial}(l)$, and $\left(p k_{1}, s k_{1}\right), \ldots,\left(p k_{n}, s k_{n}\right)$ are generated independently according to $\operatorname{Gen}\left(1^{l}\right)$ where $p k_{i}$ and $s k_{i}$ are a user $i$ 's public key and secret key, respectively. Then, we call probabilistic Batch a batch verification algorithm when the following conditions hold.

(i) If Verify $\left(\sigma_{i}, m_{i}, p k_{i}\right)=1$ for all $i \in[1, n]$, then $\operatorname{Batch}\left(\left(\sigma_{1}, m_{1}, p k_{1}\right), \ldots,\left(\sigma_{n}, m_{n}, p k_{n}\right)\right)=1$ where $m_{i}$ and $\sigma_{i}$ are a message and a signature, respectively. (ii) If Verify $\left(\sigma_{i}, m_{i}, p k_{i}\right)=0$ for some $i \in[1, n]$, then $\operatorname{Batch}\left(\left(\sigma_{1}, m_{1}, p k_{1}\right), \ldots,\left(\sigma_{n}, m_{n}, p k_{n}\right)\right)=1$ with probability negligible in $k$, taken over the randomness of Batch.

Definition 2 (a certificateless signature scheme with batch verification). A certificateless signature scheme with batch verification consists of the following algorithms.

(i) Setup: PKG randomly chooses a secret key and computes the public key $T_{\text {pub }}$ by using the secret key. Then, it publishes $T_{\text {pub }}$ and other system parameters.

(ii) KeyGen: a user $I D_{i}$ first randomly chooses a secret key $x_{i}$, computes corresponding public key $P_{i}$ and then sends $P_{i}$ to PKG. After receiving $P_{i}$, PKG outputs a partial private key $D_{i}$ to a legal user with identity $I D_{i}$.

(iii) Signing $\left(P_{i}, D_{i}, x_{i}, m\right)$ : this algorithm gets a user's public key $P_{i}$, the user's partial private key $D_{i}$, the user's secret key $x_{i}$, and a message $m$ and then it outputs a signature $\sigma$ on $m$.

(iv) Verifying $\left(\sigma, m, I D_{i}, P_{i}\right)$ : this algorithm gets a signature $\sigma$ on a message $m$, a signer's identity $I D_{i}$, and a signer's public key $P_{i}$. It then outputs True or False.

(v) Batch_Verify $\left(\left(\sigma_{1}, m_{1}, I D_{1}, P_{1}\right), \ldots,\left(\sigma_{n}, m_{n}, I D_{n}, P_{n}\right)\right)$ : this algorithm gets $n$ signatures $\sigma_{1}, \ldots, \sigma_{n}$ on message $m_{1}, \ldots, m_{n}$, the signers' identities $I D_{1}, \ldots, I D_{n}$, and the signers' public keys $P_{1}, \ldots, P_{n}$, respectively. This algorithm outputs True or False.

Definition 3 (Girault's security [19, 20]). Girault proposed three security levels to classify the levels of trust to PKG. The three levels are described as follows.

(i) Level 1: PKG knows the secret of any user.

(ii) Level 2: PKG cannot find out all the information of a user's secret. However, PKG can generate a contradictory public key (or a contradictory certificate) and impersonate the user to generate signatures with respect to the contradictory public key.

(iii) Level 3: PKG cannot find out all the information of a user's secret nor generate a contradictory public key. PKG can only generate a valid public key (or a valid certificate).

Definition 4 (the computational Diffie-Hellman (CDH) problem). Let $G_{1}$ be a cyclic group of order $q$ and let $P$ be a generator of $G_{1}$. Given $\left\langle G_{1}, q, P, a P, b P\right\rangle$ for some $a, b \in Z_{q}^{*}$, compute $a b P$.

\section{Our Proposed Scheme}

In this section, we propose an efficient certificateless signature scheme with batch verification based on [15]. $G_{1}$ is an additive group and $G_{2}$ is a multiplicative group. Let $G_{1}$ and $G_{2}$ be two cyclic groups of prime order $q$. Let $P$ be a randomly chosen generator of $G_{1}$ and $e$ a bilinear mapping such that 
$e: G_{1} \times G_{1} \rightarrow G_{2}$. The details of our scheme are described as follows.

Setup. PKG performs the following operations.

(1) Choose an integer $\lambda \in Z_{q}^{*}$ and $\theta \in\{0,1\}^{*}$ randomly, and set $T_{\text {pub }}=\lambda P$.

(2) Choose four cryptographic one-way hash functions, $H_{0}:\{0,1\}^{*} \rightarrow G_{1}, H_{1}: G_{1} \rightarrow G_{1}, H_{2}:\{0,1\}^{*} \times$ $G_{1} \times G_{1} \rightarrow Z_{q}^{*}, H_{3}:\{0,1\}^{*} \times G_{1} \times G_{1} \rightarrow Z_{q}^{*}$, and $H_{4}:\{0,1\}^{*} \rightarrow G_{1}$.

(3) Publish the system parameters $\left\{G_{1}, G_{2}, e, q, P, \theta, T_{\text {pub }}\right.$, $H_{0}, H_{1}, H_{2}, H_{3}, H_{4}$ \}and keep the master key $\lambda$ secret.

\section{Key Generating Phase}

(1) A user with identity $I D_{i}$ randomly chooses $x_{i} \in Z_{q}^{*}$ and computes $P_{i}=x_{i} P$, where $x_{i}$ and $P_{i}$ are called the secret key and the public key, respectively, of user $I D_{i}$.

(2) The user sends $\left(I D_{i}, P_{i}\right)$ to PKG.

(3) PKG gets $Q_{i}=H_{0}\left(I D_{i}\right)$ and $\Gamma_{i}=H_{1}\left(P_{i}\right)$.

(4) PKG computes $D_{i_{0}}=\lambda Q_{i}$ and $D_{i_{1}}=\lambda \Gamma_{i}$ and sends $\left(D_{i_{0}}, D_{i_{1}}\right)$ to the user via a secret channel. The pair $\left(D_{i_{0}}, D_{i_{1}}\right)$ is called the partial private key of user $I D_{i}$. The private key of user $I D_{i}$ consists of $x_{i}$ and $\left(D_{i_{0}}, D_{i_{1}}\right)$.

Signing Phase. Assume that a signer $I D_{i}$ wants to sign a message $m \in\{0,1\}^{*}$. The signer does the following works.

(1) Choose $r$ and $\alpha \in Z_{q}^{*}$ randomly.

(2) Compute $U_{1}=r\left(Q_{i}+\Gamma_{i}\right)$ and $U_{2}=\alpha x_{i} P$.

(3) Compute $h_{2}=H_{2}\left(m, U_{1}, U_{2}\right), h_{3}=H_{3}\left(m, U_{2}, U_{1}\right)$, and $W=H_{4}(\theta)$.

(4) Compute $V=\left(r+h_{2}\right)\left(D_{i_{0}}+D_{i_{1}}\right)+\left(\alpha+h_{3}\right) x_{i} W$.

(5) The signature on $m$ is $\sigma=\left(V, U_{1}, U_{2}, P_{i}\right)$.

Verifying Phase. To verify a signature $\left(V, U_{1}, U_{2}, P_{i}\right)$ on message $m$, a verifier should do the following works.

(1) Compute $Q_{i}=H_{0}\left(I D_{i}\right), \Gamma_{i}=H_{1}\left(P_{i}\right), h_{2}=$ $H_{2}\left(m, U_{1}, U_{2}\right), h_{3}=H_{3}\left(m, U_{2}, U_{1}\right)$, and $W=H_{4}(\theta)$.

(2) Verify if $e(P, V)=e\left(T_{\text {pub }}, U_{1}+h_{2}\left(Q_{i}+\Gamma_{i}\right)\right) e\left(W, U_{2}+\right.$ $\left.h_{3} P_{i}\right)$.

If it is true, the verifier accepts the signature; otherwise, the verifier rejects it.

Batch Verifying Phase. To verify $n$ signatures $\sigma_{1}=$ $\left(V_{1}, U_{1_{1}}, U_{2_{1}}, P_{1}\right), \ldots, \sigma_{n}=\left(V_{n}, U_{1_{n}}, U_{2_{n}}, P_{n}\right)$ of the $n$ signers $I D_{1}, \ldots, I D_{n}$ on message $m_{1}, \ldots, m_{n}$, respectively, a verifier performs the following works.
(1) Choose $w_{1}, \ldots, w_{n} \in Z_{q}^{*}$ randomly.

(2) Compute $Q_{i}=H_{0}\left(I D_{i}\right), \Gamma_{i}=H_{1}\left(P_{i}\right), h_{2}=H_{2}\left(m_{i}\right.$, $\left.U_{1_{i}}, U_{2_{i}}\right), h_{3_{i}}=H_{3}\left(m_{i}, U_{2_{i}}, U_{1_{i}}\right)$ for $i=1, \ldots, n$, and $W=H_{4}(\theta)$.

(3) Verify if $e\left(P, \sum_{i=1}^{n} w_{i} V_{i}\right)=e\left(T_{\text {pub }}, \sum_{i=1}^{n} w_{i} U_{1_{i}}+\right.$ $\left.w_{i} h_{2_{i}}\left(Q_{i}+\Gamma_{i}\right)\right) e\left(W, \sum_{i=1}^{n} w_{i} U_{2_{i}}+w_{i} h_{3_{i}} P_{i}\right)$.

If it is true, the verifier accepts the $n$ signatures.

Correctness. Consider

$$
\begin{aligned}
e\left(P, \sum_{i=1}^{n} w_{i} V_{i}\right) & \\
= & e\left(P, \sum_{i=1}^{n} w_{i}\left(\left(r_{i}+h_{2_{i}}\right)\left(D_{i_{0}}+D_{i_{1}}\right)\right.\right. \\
= & \left.e\left(P, \sum_{i=1}^{n} w_{i}\left(r_{i}\left(D_{i_{0}}+h_{3_{i}}\right) x_{i_{1}}\right)+h_{2_{i}}\left(D_{i_{0}}+D_{i_{1}}\right)\right)\right) \\
& \times e\left(P, \sum_{i=1}^{n} w_{i}\left(\alpha_{i} x_{i} W+h_{3_{i}} x_{i} W\right)\right) \\
= & e\left(\lambda P, \sum_{i=1}^{n} w_{i}\left(r_{i}\left(Q_{i}+\Gamma_{i}\right)+h_{2_{i}}\left(Q_{i}+\Gamma_{i}\right)\right)\right) \\
= & e\left(T \text { pub } \sum_{i=1}^{n} w_{i}\left(U_{1_{i}}+h_{2_{i}}\left(Q_{i}+\Gamma_{i}\right)\right)\right) \\
& \times e\left(W, \sum_{i=1}^{n} w_{i}\left(\alpha_{i} x_{i} P+h_{3_{i}} x_{i} P\right)\right) \\
& \left.\times\left(w_{i}+h_{3_{i}} P_{i}\right)\right) .
\end{aligned}
$$

\section{Security Models and Formal Proofs}

4.1. Security Models. A simulator $B$ simulates an environment such that an adversary $E$ can query signatures from $B$. If $E$ can forge a signature, $B$ can use the output from $E$ to solve a hard problem.

We classify adversary $E$ into three types. The adversary of type I cannot access the master secret key and query the partial private key of target ID. The adversary of type II can access the master secret key but cannot query target ID's secret key nor replace her/his public key. The adversary of type III is to simulate the environment for the proof of that a user cannot produce a signature with a new public-secret key pair, which is different from his own one, without the corresponding partial private key generated from the master secret key.

We define the capability of $E$ which can be captured by the following queries. 
(i) $H_{0}\left(I D_{i}\right)$ : if $E$ inputs a user's identity $I D_{i}$ to $H_{0}, B$ will output a randomly chosen $Q_{i} \in G_{1}$ as the user's public key.

(ii) $H_{1}\left(P_{i}\right)$ : when $E$ inputs a public key $P_{i} \in G_{1}$ to $H_{1}, B$ outputs a randomly chosen $\Gamma_{i} \in G_{1}$.

(iii) $H_{2}\left(m, U_{1}, U_{2}\right)$ : if $E$ inputs a message $m \in\{0,1\}^{*}$ and $U_{1}, U_{2} \in G_{1}, B$ will output an integer randomly chosen in $Z_{q}^{*}$.

(iv) $H_{3}\left(m, U_{2}, U_{1}\right)$ : if $E$ inputs a message $m \in\{0,1\}^{*}, U_{2} \in$ $G_{1}$, and $U_{1} \in G_{1}, B$ will output a random integer in $Z_{q}^{*}$.

(v) $H_{4}(\varrho)$ : if $E$ inputs a string $\varrho \in\{0,1\}^{*}, B$ will output an element randomly chosen in $G_{1}$.

(vi) Public $K e y\left(I D_{i}\right)$ : if $E$ inputs a user's identity, $I D_{i}$, then $B$ will output the user's public key $P_{i}$.

(vii) Partial_Private_Key $\left(I D_{i}, P_{i}\right)$ : if $E$ inputs a user's identity $I D_{i}$ and the user's public key $P_{i}, B$ will output the partial private key $\left(D_{i_{0}}, D_{i_{1}}\right)$.

(viii) Secret $K e y\left(I D_{i}\right)$ : if $E$ inputs a user's identity, $I D_{i}, B$ will output the secret key $x_{i}$ of user $I D_{i}$ to $E$.

(ix) Public_Key_Replacement $\left(I D_{i}, P_{i}^{\prime}\right)$ : when $E$ inputs a user's identity $I D_{i}$ and the user's new public key $P_{i}^{\prime}, B$ will replace $P_{i}$ with $P_{i}^{\prime}$. The new partial private key can be obtained by querying Partial_Private_Key $\left(I D_{i}\right)$.

(x) $\operatorname{Sign}\left(I D_{i}, m\right)$ : if $E$ inputs a user's identity $I D_{i}$ and a message $m, B$ will output a user $I D_{i}$ 's signature $\sigma$ on $m$ to $E$.

Definition 5 (the $\mathrm{CDH}$ assumption). We say that the $(t, \varepsilon)$ $\mathrm{CDH}$ assumption holds in $G_{1}$ if no polynomial-time algorithm within running time $t$ can solve the $\mathrm{CDH}$ problem with probability at least $\varepsilon$.

Definition 6 (the unforgeability game I). Let $E_{1}$ be a polynomial-time attacker of type I. $E_{1}$ interacts with a challenger $B$ in the following game.

(i) Step 1: $B$ runs the setup algorithm of a certificateless signature scheme with batch verification. $B$ publishes the public parameters.

(ii) Step 2: $E_{1}$ queries Partial_Private_Key, Public_Key, Public_Key_Replacement, Secret_Key, Sign, $H_{0}, H_{1}$, $\mathrm{H}_{2}, \mathrm{H}_{3}$, and $\mathrm{H}_{4}$ in an arbitrary sequence.

(iii) Step 3: $E_{1}$ outputs $n$ signatures $\sigma_{1}, \ldots, \sigma_{n}$ on $m_{1}, \ldots, m_{n}$ corresponding to the signers $I D_{1}, \ldots, I D_{n}$ with the public keys $P_{1}, \ldots, P_{n}$, respectively.

$E_{1}$ wins the game if

(1) Batch_Verify $\left(\left(\sigma_{1}, m_{1}, I D_{1}, P_{1}\right), \ldots,\left(\sigma_{n}, m_{n}, I D_{n}, P_{n}\right)\right)$ = True;

(2) there exists $\sigma_{y} \in\left\{\sigma_{1}, \ldots, \sigma_{n}\right\}$ whose $\left(I D_{y}, m_{y}\right)$ has not been queried to Sign oracle;

(3) Partial_Private_Key $\left(I D_{y}, P_{y}\right)$ has never been queried.
The scheme is $(t, \varepsilon, \mathrm{I})$-unforgeable if no polynomialtime attacker $E_{1}$, with running time at most $t$, can win the unforgeability game I with probability at least $\varepsilon$.

Definition 7 (the unforgeability game II). Let $E_{2}$ be a polynomial-time attacker of type II. $E_{2}$ interacts with a challenger $B$ in the following game.

(i) Step 1: $B$ runs the setup algorithm of a certificateless signature scheme with batch verification. $B$ publishes the public parameters and sends the master secret key to $E_{2}$.

(ii) Step 2: $E_{2}$ queries Partial_Private_Key, Public_Key, Public_Key_Replacement, Secret_Key,Sign, $H_{0}, H_{1}$, $\mathrm{H}_{2}, \mathrm{H}_{3}$, and $\mathrm{H}_{4}$ in an arbitrary sequence.

(iii) Step 3: $E_{2}$ outputs $n$ signatures $\sigma_{1}, \ldots, \sigma_{n}$ on $m_{1}, \ldots, m_{n}$ corresponding to the signers $I D_{1}, \ldots, I D_{n}$ with the public keys $P_{1}, \ldots, P_{n}$, respectively.

$E_{2}$ wins the game if

(1) Batch_Verify $\left(\left(\sigma_{1}, m_{1}, I D_{1}, P_{1}\right), \ldots,\left(\sigma_{n}, m_{n}, I D_{n}, P_{n}\right)\right)$ $=$ True;

(2) there exists $\sigma_{y} \in\left\{\sigma_{1}, \ldots, \sigma_{n}\right\}$ whose $\left(I D_{y}, m_{y}\right)$ has not been queried to Sign oracle;

(3) Neither Secret_Key $\left(I D_{y}\right)$ nor Public_Key_Replacement $\left(I D_{y}, \cdot, \cdot\right)$ has been queried.

The scheme is $(t, \varepsilon, \mathrm{II})$-unforgeable if no polynomialtime attacker $E_{2}$, with running time at most $t$, can win the unforgeability game II with probability at least $\varepsilon$.

Definition 8 (the unforgeability game III [19]). Let $E_{3}$ be a polynomial-time attacker of type III. $E_{3}$ interacts with a challenger $B$ in the following game.

(I) Step 1: $B$ runs the setup algorithm of a certificateless signature scheme with batch verification. $B$ publishes the public parameters.

(II) Step 2: $E_{3}$ queries Partial_Private_Key, Public_Key, Public_Key_Replacement, Secret_Key, $\mathrm{H}_{0}, \mathrm{H}_{1}, \mathrm{H}_{2}$, $\mathrm{H}_{3}$, and $\mathrm{H}_{4}$ in an arbitrary sequence.

(III) Step 3: $E_{3}$ outputs a signature $\sigma^{*}=\left(V^{*}, U_{1}^{*}, U_{2}^{*}, P_{y}^{*}\right)$ on a message $m^{*}$ for the user $I D_{y}$.

$E_{3}$ wins the game if

(1) Verifying $\left(\sigma^{*}, m^{*}, I D_{y}, P_{y}^{*}\right)=$ True;

(2) User $I D_{y}$ has been created, that is, Public_Key $\left(I D_{y}\right)$, Partial_Private_Key $\left(I D_{y}, P_{y}\right)$, and Secret_Key $\left(I D_{y}\right)$ have been queried;

(3) $P_{y}^{*}$ is different from all of the public keys $P_{y}$ 's returned by Public_Key $\left(I D_{y}\right)$ or used to query Public_Key_Replacement.

The scheme is $(t, \varepsilon$, III)-unforgeable if no polynomialtime attacker $E_{3}$, with running time at most $t$, can win the unforgeability game III with probability at least $\varepsilon$. 
4.2. Formal Proofs. In this section, we will prove that our scheme is unforgeable based on the $\mathrm{CDH}$ assumption.

Lemma 9 (the forking lemma [40]). Let $(s, m, c)$ be a valid signature-message triple of a signature scheme and $h$ the hashed value of $(m, c)$ where $m$ is a plaintext message, $c$ is a string, and $s$ is called the signature part of the triple. Let $A$ be $a$ probabilistic polynomial-time Turning machine. Given only the public data of the signature scheme as input, if A can find, with nonnegligible probability, a valid signature-message triple $(s, m, c)$ with $h$, then, with nonnegligible probability, a replay of this machine, with the same random tape and a different value returned by the random oracle, outputs two valid signaturemessage triples $(s, m, c)$ with $h$ and $\left(s^{\prime}, m, c\right)$ with $h^{\prime}$ such that $h \neq h^{\prime}$.

Lemma 10 (the splitting lemma [40]). Let $A \subset X \times Y$ such that $\operatorname{Pr}[(x, y) \in A] \geq \varepsilon$. For any $\alpha<\varepsilon$, define $B=\{(x, y) \in$ $\left.X \times Y \mid \operatorname{Pr}_{y^{\prime} \in Y}\left[\left(x, y^{\prime}\right) \in A\right] \geq \varepsilon-\alpha\right\}$ and then the following statements hold:

(1) $\operatorname{Pr}[B] \geq \alpha$,

(2) $\forall(x, y) \in B, \operatorname{Pr}_{y^{\prime} \in Y}\left[\left(x, y^{\prime}\right) \in A\right] \geq \varepsilon-\alpha$,

(3) $\operatorname{Pr}[B \mid A] \geq \alpha / \varepsilon$.

Theorem 11. Given $n$ 5-tuples $\left(V_{i}, U_{1_{i}}, U_{2_{i}}, P_{i}, m_{i}\right)$ 's, if $e(P$, $\left.\sum_{i=1}^{n} w_{i} V_{i}\right)=e\left(T_{p u b}, \sum_{i=1}^{n} w_{i} U_{1_{i}}+w_{i} h_{2_{i}}\left(Q_{i}+\Gamma_{i}\right)\right) e(W$, $\left.\sum_{i=1}^{n} w_{i} U_{2_{i}}+w_{i} h_{3_{i}} P_{i}\right)$ where $w_{i}$ is randomly chosen in $Z_{q}^{*}$, $h_{2_{i}}=H_{2}\left(m_{i}, U_{1_{1}}, U_{2_{i}}\right)$, and $h_{3_{i}}=H_{3}\left(m_{i}, U_{2_{i}}, U_{1_{i}}\right)$ for each $i$, the probability of that $e\left(P, V_{i}\right) \neq e\left(T_{\text {pub }}, U_{1_{i}}+h_{2_{i}}\left(Q_{i}+\Gamma_{i}\right)\right) e\left(W, U_{2_{i}}+\right.$ $\left.h_{3_{i}} P_{i}\right)$ for some $i \in\{1, \ldots, n\}$ is $1 / 2^{|q|}$.

Proof. The proof is based on [35, 37]. If $e\left(P, V_{i}\right) \neq e\left(T_{\text {pub }}, U_{1_{i}}+\right.$ $\left.h_{2_{i}}\left(Q_{i}+\Gamma_{i}\right)\right) e\left(W, U_{2_{i}}+h_{3_{i}} P_{i}\right)$ for some $i$, we have that $V_{i} \neq\left(r_{i}+\right.$ $\left.h_{2_{i}}\right)\left(\lambda Q_{i}+\lambda \Gamma_{i}\right)+\left(\alpha_{i}+h_{3_{i}}\right) x_{i} W$ for some $i$. Thus, there exists $c_{i} \neq 0(\bmod q)$ such that $V_{i}=\left(r_{i}+h_{2_{i}}\right)\left(\lambda Q_{i}+\lambda \Gamma_{i}\right)+\left(\alpha_{i}+\right.$ $\left.h_{3_{i}}\right) x_{i} W+c_{i} P$ for some $i$.

Let $V_{j}=\left(r_{j}+h_{2_{j}}\right)\left(\lambda Q_{j}+\lambda \Gamma_{j}\right)+\left(\alpha_{j}+h_{3_{j}}\right) x_{j} W+c_{j} P$ and $c_{j} \in\{0,1, \ldots, q-1\}, \forall j \in\{1, \ldots, n\}-\{i\}$. As $e\left(P, \sum_{i=1}^{n} w_{i} V_{i}\right)=$ $e\left(T_{\text {pub }}, \sum_{i=1}^{n} w_{i} U_{1_{i}}+w_{i} h_{2_{i}}\left(Q_{i}+\Gamma_{i}\right)\right) e\left(W, \sum_{i=1}^{n} w_{i} U_{2_{i}}+w_{i} h_{3_{i}} P_{i}\right)$, $w_{1} c_{1}+w_{2} c_{2}+w_{3} c_{3}+\cdots+w_{n} c_{n} \equiv 0(\bmod q)$ and thus $w_{i}=$ $c_{i}^{-1}\left(w_{1} c_{1}+w_{2} c_{2}+\cdots+w_{i-1} c_{i-1}+w_{i+1} c_{i+1}+\cdots+w_{n} c_{n}\right) \bmod$ $q$. Since $w_{i}$ is randomly chosen in $Z_{q}^{*}$, the probability of $w_{i}=$ $c_{i}^{-1}\left(w_{1} c_{1}+w_{2} c_{2}+\cdots+w_{i-1} c_{i-1}+w_{i+1} c_{i+1}+\cdots+w_{n} c_{n}\right) \bmod q$ is $1 / 2^{|q|}$, which is negligible.

Theorem 12. The proposed scheme is $\left(t, q_{s i g n}, q_{p p k}, q_{s k}, q_{p k r}\right.$, $\left.q_{p k}, q_{h_{0}}, q_{h_{1}}, q_{h_{2}}, q_{h_{3}}, q_{h_{4}}, \varepsilon, I\right)$-unforgeable assuming that the $\left(t^{\prime}, \varepsilon^{\prime}\right)-C D H$ assumption holds in $G_{1}$ where $\varepsilon^{\prime} \geq\left(\varepsilon /\left(2 q_{h_{0}}\right)\right)$ $\left(1-1 / 2^{|q|}\right)^{2}, t^{\prime} \approx t+q_{s i g n} \mathcal{O}\left(t_{s i g n}\right)+q_{p p k} \mathcal{O}\left(t_{p p k}\right)+q_{s k} \mathcal{O}\left(t_{s k}\right)+$ $q_{p k r} \mathcal{O}\left(t_{p k r}\right)+q_{p k} \mathcal{O}\left(t_{p k}\right)+q_{h_{0}} \mathcal{O}\left(t_{h_{0}}\right)+q_{h_{1}} \mathcal{O}\left(t_{h_{1}}\right)+q_{h_{2}} \mathcal{O}\left(t_{h_{2}}\right)+$ $q_{h_{3}} \mathcal{O}\left(t_{h_{3}}\right)+q_{h_{4}} \mathcal{O}\left(t_{h_{4}}\right)+\mathcal{O}(1), q_{s i g n}, q_{p p k}, q_{s k}, q_{p k r}, q_{p k}, q_{h_{0}}$, $q_{h_{1}}, q_{h_{2}}, q_{h_{3}}$, and $q_{h_{4}}$ being the numbers of queries to Sign, Partial_Private_Key, Secret_Key, Public_Key_Replacement, Public_Key, $H_{0}, H_{1}, H_{2}, H_{3}$, and $H_{4}$, respectively, and $t_{\text {sign }}$, $t_{p p k}, t_{s k}, t_{p k r}, t_{p k}, t_{h_{0}}, t_{h_{1}}, t_{h_{2}}, t_{h_{3}}$, and $t_{h_{4}}$ being the computing time of the queries to Sign, Partial_Private_Key, Secret_Key,
Public_Key_Replacement, Public_Key, $H_{0}, H_{1}, H_{2}, H_{3}$, and $\mathrm{H}_{4}$, respectively.

Proof. Assume that a polynomial-time attacker $E_{1}$ wins the game of Definition 6 with probability being at least $\varepsilon$ within running time $t$. A simulator $B$ is given an instance of the $\mathrm{CDH}$ problem $\left\langle G_{1}, q, P, a P, b P\right\rangle$, and $B$ 's goal is to output the value of $a b P$. We will construct $B$ which plays the game in Definition 6 with $E_{1}$ and outputs the value of $a b P$.

Setup. $B$ sets $T_{\text {pub }}=b P$ and chooses $\theta \in\{0,1\}^{*}$ randomly. Then, $B$ publishes $\left\{G_{1}, G_{2}, e, q, P, \theta, T_{\text {pub }}, H_{0}, H_{1}, H_{2}, H_{3}, H_{4}\right\}$. $B$ can respond to the queries from $E_{1}$ as follows.

(i) $H_{0}$ query: $B$ constructs a list, $H_{0}$-list, and chooses an identity $I D_{\pi}$ randomly. When $E_{1}$ queries $H_{0}\left(I D_{i}\right)$ to $B, B$ checks whether $I D_{i}$ is in $H_{0}$-list or not. If $I D_{i}$ does not exist in $\mathrm{H}_{0}$-list, then there are the following two cases. Case 1: if $I D_{i}=I D_{\pi}, B$ sets $Q_{i}=a P=H_{0}\left(I D_{i}\right)$ and stores $\left(I D_{i}, a P\right)$ in $H_{0}$-list. Case 2: if $I D_{i} \neq I D_{\pi}, B$ sets $Q_{i}=k_{i} P=H_{0}\left(I D_{i}\right)$ where $k_{i}$ is randomly chosen in $Z_{q}^{*}$ and stores $\left(I D_{i}, k_{i} P, k_{i}\right)$ in $H_{0}$-list. However, if $I D_{i}$ exists in $H_{0}$-list, $B$ gets its mapping value, $Q_{i}=$ $k_{i} P$ or $a P$. Finally, $B$ returns $Q_{i}$.

(ii) $H_{1}$ query: $B$ constructs a list, $H_{1}$-list. If $E_{1}$ queries $H_{1}\left(P_{i}\right)$ to $B$ where $P_{i} \in G_{1}, B$ checks whether $P_{i}$ is in $H_{1}$-list or not. If it does not exist in $H_{1}$-list, $B$ randomly chooses $\rho_{i} \in Z_{q}^{*}$ and records $\left(P_{i}, \rho_{i} P, \rho_{i}\right)$ in $H_{1}$-list; else, $B$ gets its mapping value, $\rho_{i} P$, from $H_{1}$ list. Then, $B$ returns $\rho_{i} P$ to $E_{1}$.

(iii) $\mathrm{H}_{2}$ query: $B$ constructs $H_{2}$-list. If $E_{1}$ queries $H_{2}\left(m, U_{1}, U_{2}\right)$ to $B, B$ checks whether $\left(m, U_{1}, U_{2}\right)$ is in $\mathrm{H}_{2}$-list or not. If not, $B$ randomly chooses $h_{2} \in Z_{q}^{*}$ and records $\left(\left(m, U_{1}, U_{2}\right), h_{2}\right)$ in $H_{2}$-list; else, $B$ gets its mapping value, $h_{2}$, from $H_{2}$-list. Then, $B$ returns $h_{2}$ to $E_{1}$.

(iv) $H_{3}$ query: $B$ constructs $H_{3}$-list. When $E_{1}$ queries $H_{3}\left(m, U_{2}, U_{1}\right)$ to $B, B$ checks whether $\left(m, U_{2}, U_{1}\right)$ is in $H_{3}$-list or not. If not, $B$ randomly chooses $h_{3} \in Z_{q}^{*}$ and records $\left(\left(m, U_{2}, U_{1}\right), h_{3}\right)$ in $H_{3}$-list; otherwise, $B$ gets its mapping value, $h_{3}$, from $\mathrm{H}_{3}$-list. Then, $B$ responds $h_{3}$ to $E_{1}$.

(v) $H_{4}$ query: $B$ constructs $H_{4}$-list. If $E_{1}$ queries $H_{4}$ with a string $\varrho$ to $B, B$ checks whether $\varrho$ is in $H_{4}$-list or not. If $\varrho$ does not exist in $\mathrm{H}_{4}$-list, then there are the following two conditions. Condition 1 : if $\varrho=\theta, B$ sets $W=\beta P=$ $H_{4}(\varrho)$ where $\beta$ is randomly chosen in $Z_{q}^{*}$ and stores $(\varrho, \beta P, \beta)$ in $H_{4}$-list. Condition 2: if $\varrho \neq \theta, B$ sets $W=$ $\Phi=H_{4}(\varrho)$ where $\Phi$ is randomly chosen in $G_{1}$ and stores $(\varrho, \Phi)$ in $\mathrm{H}_{4}$-list. However, if $\varrho$ exists in $\mathrm{H}_{4}$-list, $B$ gets its mapping value, $W=\Phi$ or $\beta P$. Finally, $B$ returns $W$.

(vi) Public_Key query: $B$ constructs a list, $p k$-list. When $E_{1}$ queries Public_Key $\left(I D_{i}\right)$ to $B, B$ looks up pk-list. If $I D_{i}$ is not found in $p k$-list, $B$ randomly chooses $x_{i} \in$ 
$Z_{q}^{*}$, computes $P_{i}=x_{i} P$, and stores $\left(I D_{i}, P_{i}, x_{i}\right)$ in $p k$ list; otherwise, $B$ gets $P_{i}$ from $p k$-list. Finally, $B$ returns $P_{i}$.

(vii) Partial_Private_Key query: if $E_{1}$ queries Partial Private_Key $\left(I D_{i}, P_{i}^{\prime}\right)$ to $B, B$ looks up $H_{0}$-list, $p k$-list, and $H_{1}$-list. If $I D_{i}=I D_{\pi}, B$ returns "failure." If $I D_{i}$ is not found in $H_{0}$-list, $B$ queries $H_{0}\left(I D_{i}\right)$ and gets $k_{i}$ from $H_{0}$-list; else, $B$ retrieves $k_{i}$ from $H_{0}$-list. If $I D_{i}$ is not in $p k$-list, $B$ queries Public_Key $\left(I D_{i}\right)$ and obtains $P_{i}$; otherwise, $B$ catches $P_{i}$ from $p k$-list. If $P_{i} \neq P_{i}^{\prime}, B$ returns "failure." Then, if $P_{i}$ is not found in $H_{1}$-list, $B$ queries $H_{1}\left(P_{i}\right)$ and gets $\rho_{i}$; else, $B$ retrieves $\rho_{i}$ from $H_{1}$ list. Finally, $B$ returns $\left(D_{i_{0}}=k_{i} b P, D_{i_{1}}=\rho_{i} b P\right)$.

(viii) Secret_Key query: if $E_{1}$ queries Secret_Key $\left(I D_{i}\right)$ to $B, B$ looks up $p k$-list. If $I D_{i}$ is not found in $p k$-list, $B$ queries Public_Key $\left(I D_{i}\right)$ and gets $x_{i}$; if there is a record $\left(I D_{i}, P_{i}, x_{i}\right)$, then $B$ retrieves $x_{i}$ from $p k$-list and returns $x_{i}$.

(ix) Public_Key_Replacement query: when $E_{1}$ queries Public_Key_Replacement $\left(I D_{i}, P_{i}^{\prime}\right)$ to $B, B$ looks up $p k$-list. If $I D_{i}$ is not found in $p k$-list, $B$ queries Public Key $\left(I D_{i}\right)$. Then, $B$ replaces the record $\left(I D_{i}, P_{i}, x_{i}\right)$ with $\left(I D_{i}, P_{i}^{\prime}, \perp\right)$ in $p k$-list.

(x) Sign query: when $E_{1}$ queries $\operatorname{Sign}\left(I D_{i}, m\right)$ to $B$, if $I D_{i}=I D_{\pi}, B$ does the following works.

(1) Choose $z, \alpha$, and $h_{2} \in Z_{q}^{*}$ randomly;

(2) compute $U_{1}=z P-h_{2} a P$ and $U_{2}=\alpha P$;

(3) set $H_{2}\left(m, U_{1}, U_{2}\right)=h_{2}$;

(4) compute $h_{3}=H_{3}\left(m, U_{2}, U_{1}\right)$ and $W=H_{4}(\theta)$;

(5) compute $V=z b P+h_{2} \rho_{i} b P+\alpha \beta P+h_{3} \beta P_{i}$;

(6) form $\sigma=\left(V, U_{1}, U_{2}, P_{i}\right)$.

Thus, the signature on $m$ is $\sigma=\left(V, U_{1}, U_{2}, P_{i}\right)$ and it satisfies the verifying formula in Section 3.

If $I D_{i} \neq I D_{\pi}, B$ can return a signature on $m$ to $E_{1}$ because $B$ can compute all secrets of user $I D_{i}$. Finally, suppose that $E_{1}$ outputs, with probability at least $\varepsilon, n$ signatures $\sigma_{1}, \ldots, \sigma_{n}$ on $m_{1}, \ldots, m_{n}$ of the signers $I D_{1}, \ldots, I D_{n}$, respectively, such that

(1) Batch_Verify $\left(\left(\sigma_{1}, m_{1}, I D_{1}, P_{1}\right), \ldots,\left(\sigma_{n}, m_{n}, I D_{n}, P_{n}\right)\right)$ = True;

(2) there exists $\sigma_{y} \in\left\{\sigma_{1}, \ldots, \sigma_{n}\right\}$ which is not the output from $\operatorname{Sign}\left(I D_{y}, m_{y}\right)$;

(3) Partial_Private_Key $\left(I D_{y}, P_{y}\right)$ has never been queried.

From Lemma 9, we fork the sequence of signatures one time and get $\sigma_{1}^{\prime}, \ldots, \sigma_{n}^{\prime}$ on $m_{1}, \ldots, m_{n}$ by setting $h_{2_{y}}^{\prime} \neq h_{2_{y}}$.
Thus, we randomly choose $w_{i}$ 's and obtain the following two equations:

$$
\begin{aligned}
e\left(P, \sum_{i=1}^{n} w_{i} V_{i}\right) & \\
= & e\left(T_{\mathrm{pub}}, \sum_{i=1}^{n} w_{i} U_{1_{i}}+w_{i} h_{2_{i}}\left(Q_{i}+\Gamma_{i}\right)\right) \\
& \times e\left(W, \sum_{i=1}^{n} w_{i} U_{2_{i}}+w_{i} h_{3_{i}} P_{i}\right) \\
e\left(P, \sum_{i=1}^{n} w_{i} V_{i}^{\prime}\right) & \\
= & e\left(T_{\mathrm{pub}}, \sum_{i=1}^{n} w_{i} U_{1_{i}}^{\prime}+w_{i} h_{2_{i}}^{\prime}\left(Q_{i}+\Gamma_{i}\right)\right) \\
& \times e\left(W, \sum_{i=1}^{n} w_{i} U_{2_{i}}^{\prime}+w_{i} h_{3_{i}}^{\prime} P_{i}\right)
\end{aligned}
$$

with probability being at least $\varepsilon / 2$ by Lemma 10 , where $V_{y} \neq V_{y}^{\prime}, U_{1_{y}}=U_{1_{y}}^{\prime}, h_{2 y} \neq h_{2_{y}}^{\prime}, U_{2_{y}}=U_{2_{y}}^{\prime}$, and $h_{3 y}=h_{3_{y}}^{\prime}$.

We assume that $I D_{y}=I D_{\pi}$. By Theorem 11, we have that

$$
\begin{aligned}
e\left(P, V_{y}\right)= & e\left(T_{\text {pub }}, U_{1_{y}}+h_{2_{y}}\left(Q_{y}+\Gamma_{y}\right)\right) \\
& \times e\left(W, U_{2_{y}}+h_{3_{y}} P_{y}\right) \\
e\left(P, V_{y}^{\prime}\right)= & e\left(T_{\text {pub }}, U_{1_{y}}+h_{2_{y}}^{\prime}\left(Q_{y}+\Gamma_{y}\right)\right) \\
& \times e\left(W, U_{2_{y}}+h_{3_{y}} P_{y}\right)
\end{aligned}
$$

with probability being at least $\left(\varepsilon /\left(2 q_{h_{0}}\right)\right)\left(1-1 / 2^{|q|}\right)^{2}$. Thus, we can compute $\left(h_{2 y}-h_{2_{y}}^{\prime}\right)^{-1}\left(\left(V_{y}-V_{y}^{\prime}\right)-\rho_{y} T_{\text {pub }}\right)$, which is $a b P$, to solve the $\mathrm{CDH}$ problem with $\varepsilon^{\prime} \geq\left(\varepsilon /\left(2 q_{h_{0}}\right)\right)\left(1-1 / 2^{|q|}\right)^{2}$ and $t^{\prime} \approx t+q_{\text {sign }} \mathcal{O}\left(t_{\text {sign }}\right)+q_{p p k} \mathcal{O}\left(t_{p p k}\right)+q_{s k} \mathcal{O}\left(t_{s k}\right)+q_{p k r} \mathcal{O}\left(t_{p k r}\right)+$ $q_{p k} \mathcal{O}\left(t_{p k}\right)+q_{h_{0}} \mathcal{O}\left(t_{h_{0}}\right)+q_{h_{1}} \mathcal{O}\left(t_{h_{1}}\right)+q_{h_{2}} \mathcal{O}\left(t_{h_{2}}\right)+q_{h_{3}} \mathcal{O}\left(t_{h_{3}}\right)+$ $q_{h_{4}} \mathcal{O}\left(t_{h_{4}}\right)+\mathcal{O}(1)$.

Theorem 13. The proposed scheme is $\left(t, q_{s i g n}, q_{p p k}, q_{s k}, q_{p k r}\right.$, $q_{p k}, q_{h_{0}}, q_{h_{1}}, q_{h_{2}}, q_{h_{3}}, q_{h_{4}}, \varepsilon$, II)-unforgeable assuming that the $\left(t^{\prime}, \varepsilon^{\prime}\right)-C D H$ assumption holds in $G_{1}$ where $\varepsilon^{\prime} \geq\left(\varepsilon /\left(2 q_{p k}\right)\right)(1-$ $\left.1 / 2^{|q|}\right)^{2}, t^{\prime} \approx t+q_{s i g n} \mathcal{O}\left(t_{\text {sign }}\right)+q_{p p k} \mathcal{O}\left(t_{p p k}\right)+q_{s k} \mathcal{O}\left(t_{s k}\right)+$ $q_{p k r} \mathcal{O}\left(t_{p k r}\right)+q_{p k} \mathcal{O}\left(t_{p k}\right)+q_{h_{0}} \mathcal{O}\left(t_{h_{0}}\right)+q_{h_{1}} \mathcal{O}\left(t_{h_{1}}\right)+q_{h_{2}} \mathcal{O}\left(t_{h_{2}}\right)+$ $q_{h_{3}} \mathcal{O}\left(t_{h_{3}}\right)+q_{h_{4}} \mathcal{O}\left(t_{h_{4}}\right)+\mathcal{O}(1), q_{s i g n}, q_{p p k}, q_{s k}, q_{p k r}, q_{p k}, q_{h_{0}}$, $q_{h_{1}}, q_{h_{2}}, q_{h_{3}}$, and $q_{h_{4}}$ are the numbers of queries to Sign, Partial_Private_Key, Secret_Key, Public_Key_Replacement, Public_Key, $H_{0}, H_{1}, H_{2}, H_{3}$, and $H_{4}$, respectively, and $t_{\text {sign }}$, $t_{p p k}, t_{s k}, t_{p k r}, t_{p k}, t_{h_{0}}, t_{h_{1}}, t_{h_{2}}, t_{h_{3}}$, and $t_{h_{4}}$ are the computing time of the queries to Sign, Partial_Private_Key, Secret_Key, Public_Key_Replacement, Public_Key, $\mathrm{H}_{0}, \mathrm{H}_{1}, \mathrm{H}_{2}, \mathrm{H}_{3}$, and $\mathrm{H}_{4}$, respectively.

Proof. Assume that a polynomial-time attacker $E_{2}$ wins the game of Definition 7 with probability at least $\varepsilon$ within running 
time $t$. A simulator $B$ is given an instance of the $\mathrm{CDH}$ problem $\left\langle G_{1}, q, P, a P, b P\right\rangle$, and $B$ 's goal is to output the value of $a b P$. We will construct $B$ which plays the game in Definition 7 with $E_{2}$ and outputs the value of $a b P$.

Setup. $B$ sets $T_{\text {pub }}=\lambda P$ where $\lambda$ is randomly chosen in $Z_{q}^{*}$ and chooses $\theta \in\{0,1\}^{*}$ randomly. Then, $B$ publishes $\left\{G_{1}, G_{2}\right.$, $\left.e, q, P, \theta, T_{\text {pub }}, H_{0}, H_{1}, H_{2}, H_{3}, H_{4}\right\}$ and sends $\lambda$ to $E_{2}$. $B$ can respond to the queries from $E_{2}$ as follows.

(i) $H_{0}$ query: $B$ constructs a list, $H_{0}$-list. When $E_{2}$ queries $H_{0}\left(I D_{i}\right)$ to $B, B$ checks whether $I D_{i}$ is in $H_{0}$-list or not. If $I D_{i}$ does not exist in $H_{0}$-list, $B$ sets $Q_{i}=$ $k_{i} P=H_{0}\left(I D_{i}\right)$ where $k_{i}$ is a random integer in $Z_{q}^{*}$ and records $\left(I D_{i}, k_{i} P, k_{i}\right)$ in $H_{0}$-list; else, $B$ gets its mapping value, $k_{i} P$, from $H_{0}$-list. Then, $B$ returns $Q_{i}=k_{i} P$ to $E_{2}$.

(ii) $H_{4}$ query: $B$ constructs $H_{4}$-list. If $E_{2}$ queries $H_{4}$ with a string $\varrho$ to $B, B$ checks whether $\varrho$ is in $H_{4}$-list or not. If $\varrho$ does not exist in $\mathrm{H}_{4}$-list, then there are the following two conditions. Condition 1: if $\varrho=\theta, B$ sets $W=b P=H_{4}(\varrho)$ and stores $(\varrho, b P)$ in $H_{4}$-list. Condition 2: if $\varrho \neq \theta, B$ sets $W=\Phi=H_{4}(\varrho)$ where $\Phi$ is randomly chosen in $G_{1}$ and stores $(\varrho, \Phi)$ in $H_{4}$ list. However, if $\varrho$ exists in $H_{4}$-list, $B$ gets its mapping value, $W=\Phi$ or $b P$. Finally, $B$ returns $W$.

(iii) The simulations for $H_{1}, H_{2}, H_{3}$, and Public Key_Replacement are the same as those in the proof of Theorem 12

(iv) Public Key query: $B$ constructs a list, $p k$-list, and chooses an identity $I D_{\pi}$ randomly. When $E_{2}$ queries Public Key $\left(I D_{i}\right)$ to $B, B$ looks up $p k$-list. If $I D_{i}$ does not exist in $p k$-list, then there are the following two conditions. Condition 1: if $I D_{i}=I D_{\pi}, B$ sets $P_{i}=$ $a P$ and stores $\left(I D_{i}, a P\right)$ in $p k$-list. Condition 2: if $I D_{i} \neq I D_{\pi}, B$ sets $P_{i}=x_{i} P$ where $x_{i}$ is randomly chosen in $Z_{q}^{*}$ and stores $\left(I D_{i}, x_{i} P, x_{i}\right)$ in $p k$-list. However, if $I D_{i}$ exists in $p k$-list, $B$ gets its mapping value, $P_{i}=x_{i} P$ or $a P$. Finally, $B$ returns $P_{i}$.

(v) Partial_Private_Key query: when $E_{2}$ queries Partial_Private_Key $\left(I D_{i}, P_{i}^{\prime}\right)$ to $B, B$ looks up $H_{0^{-}}$ list, $p k$-list, and $H_{1}$-list. If $I D_{i}$ is not found in $H_{0}$-list, $B$ queries $H_{0}\left(I D_{i}\right)$ and gets $k_{i}$ from $H_{0}$-list; else, $B$ retrieves $k_{i}$ from $H_{0}$-list. If $I D_{i}$ is not in pk-list, $B$ queries Public $\_$Key $\left(I D_{i}\right)$ and obtains $P_{i}$; otherwise, $B$ catches $P_{i}$ from $p k$-list. If $P_{i} \neq P_{i}^{\prime}, B$ returns "failure." Then, if $P_{i}$ is not found in $H_{1}$-list, $B$ queries $H_{1}\left(P_{i}\right)$ and gets $\rho_{i}$; else, $B$ retrieves $\rho_{i}$ from $H_{1}$-list. Finally, $B$ returns $\left(D_{i_{0}}=\lambda k_{i} P, D_{i_{1}}=\lambda \rho_{i} P\right)$.

(vi) Secret $K$ Key query: when $E_{2}$ queries Secret $K e y\left(I D_{i}\right)$ to $B, B$ looks up $p k$-list. If $I D_{i}=I D_{\pi}, B$ returns "failure." If $I D_{i}$ is not found in $p k$-list, $B$ queries Public_Key $\left(I D_{i}\right)$ and gets $x_{i}$ from pk-list; else, $B$ retrieves $x_{i}$ from $p k$-list. Finally, $B$ returns $x_{i}$.

(vii) Sign query: when $E_{2}$ queries $\operatorname{Sign}\left(I D_{i}, m\right)$ to $B$, if $I D_{i}=I D_{\pi}, B$ performs the following works.
(1) choose $z, \alpha$, and $h_{3} \in Z_{q}^{*}$ randomly;

(2) compute $U_{1}=z P$ and $U_{2}=\alpha P-h_{3} a P$;

(3) compute $h_{2}=H_{2}\left(m, U_{1}, U_{2}\right)$ and $W=H_{4}(\theta)$;

(4) set $H_{3}\left(m, U_{2}, U_{1}\right)=h_{3}$;

(5) compute $V=z \lambda P+h_{2} k_{i} \lambda P+h_{2} \rho_{i} \lambda P+\alpha W$;

(6) form $\sigma=\left(V, U_{1}, U_{2}, P_{i}\right)$.

Therefore, the signature on $m$ is $\sigma=\left(V, U_{1}, U_{2}, P_{i}\right)$ and it meets the verifying formula in Section 3.

If $I D_{i} \neq I D_{\pi}, B$ can return a signature on $m$ to $E_{2}$ since $B$ knows all secrets of user $I D_{i}$. Finally, $E_{2}$ outputs, with probability being at least $\varepsilon, n$ signatures $\sigma_{1}, \ldots, \sigma_{n}$ on $m_{1}, \ldots, m_{n}$ of the signers $I D_{1}, \ldots, I D_{n}$, respectively, such that

(1) Batch_Verify $\left(\left(\sigma_{1}, m_{1}, I D_{1}, P_{1}\right), \ldots,\left(\sigma_{n}, m_{n}, I D_{n}, P_{n}\right)\right)$ = True;

(2) there exists $\sigma_{y} \in\left\{\sigma_{1}, \ldots, \sigma_{n}\right\}$ which is not the output from $\operatorname{Sign}\left(I D_{y}, m_{y}\right)$;

(3) neither Secret_Key $\left(I D_{y}\right)$ nor Public_Key_Replacement $\left(I D_{y}, \cdot, \cdot\right)$ has been queried.

From Lemma 9, we fork the sequence of signatures one time and get $\sigma_{1}^{\prime}, \ldots, \sigma_{n}^{\prime}$ on $m_{1}, \ldots, m_{n}$ by setting $h_{3_{y}}^{\prime} \neq h_{3_{y}}$. Thus, we randomly choose $w_{i}$ 's and obtain the following two equations:

$$
\begin{aligned}
e\left(P, \sum_{i=1}^{n} w_{i} V_{i}\right) & \\
= & e\left(T_{\text {pub }}, \sum_{i=1}^{n} w_{i} U_{1_{i}}+w_{i} h_{2_{i}}\left(Q_{i}+\Gamma_{i}\right)\right) \\
& \times e\left(W, \sum_{i=1}^{n} w_{i} U_{2_{i}}+w_{i} h_{3_{i}} P_{i}\right) \\
e(P, & \left.\sum_{i=1}^{n} w_{i} V_{i}^{\prime}\right) \\
= & e\left(T_{\text {pub }}, \sum_{i=1}^{n} w_{i} U_{1_{i}}^{\prime}+w_{i} h_{2_{i}}^{\prime}\left(Q_{i}+\Gamma_{i}\right)\right) \\
& \times e\left(W, \sum_{i=1}^{n} w_{i} U_{2_{i}}^{\prime}+w_{i} h_{3_{i}}^{\prime} P_{i}\right)
\end{aligned}
$$

with probability being at least $\varepsilon / 2$ by Lemma 10 , where $V_{y} \neq V_{y}^{\prime}, U_{1_{y}}=U_{1_{y}}^{\prime}, h_{2 y}=h_{2_{y}}^{\prime}, U_{2_{y}}=U_{2_{y}}^{\prime}$, and $h_{3 y} \neq h_{3_{y}}^{\prime}$.

We assume that $I D_{y}=I D_{\pi}$. By Theorem 11, we have that

$$
\begin{aligned}
e\left(P, V_{y}\right)= & e\left(T_{\text {pub }}, U_{1_{y}}+h_{2_{y}}\left(Q_{y}+\Gamma_{y}\right)\right) \\
& \times e\left(W, U_{2_{y}}+h_{3_{y}} P_{y}\right), \\
e\left(P, V_{y}^{\prime}\right)= & e\left(T_{\text {pub }}, U_{1_{y}}+h_{2_{y}}\left(Q_{y}+\Gamma_{y}\right)\right) \\
& \times e\left(W, U_{2_{y}}+h_{3_{y}}^{\prime} P_{y}\right)
\end{aligned}
$$


with probability being at least $\left(\varepsilon /\left(2 q_{p k}\right)\right)\left(1-1 / 2^{|q|}\right)^{2}$. Thus, we can compute $\left(h_{3_{y}}-h_{3_{y}}^{\prime}\right)^{-1}\left(V_{y}-V_{y}^{\prime}\right)$, which is $a b P$, to solve the CDH problem with $\varepsilon^{\prime} \geq\left(\varepsilon /\left(2 q_{p k}\right)\right)\left(1-1 / 2^{|q|}\right)^{2}$ and $t^{\prime} \approx t+q_{\text {sign }} \mathcal{O}\left(t_{\text {sign }}\right)+q_{p p k} \mathcal{O}\left(t_{p p k}\right)+q_{s k} \mathcal{O}\left(t_{s k}\right)+q_{p k r} \mathcal{O}\left(t_{p k r}\right)+$ $q_{p k} \mathcal{O}\left(t_{p k}\right)+q_{h_{0}} \mathcal{O}\left(t_{h_{0}}\right)+q_{h_{1}} \mathcal{O}\left(t_{h_{1}}\right)+q_{h_{2}} \mathcal{O}\left(t_{h_{2}}\right)+q_{h_{3}} \mathcal{O}\left(t_{h_{3}}\right)+$ $q_{h_{4}} \mathcal{O}\left(t_{h_{4}}\right)+\mathcal{O}(1)$.

Theorem 14. The proposed scheme is $\left(t, q_{p p k}, q_{s k}, q_{p k r}, q_{p k}\right.$, $q_{h_{0}}, q_{h_{1}}, q_{h_{2}}, q_{h_{3}}, q_{h_{4}}, \varepsilon$, III)-unforgeable assuming that the $\left(t^{\prime}, \varepsilon^{\prime}\right)-C D H$ assumption holds in $G_{1}$ with $\varepsilon^{\prime} \geq \varepsilon /\left(2 q_{h_{1}-\max }\right)$, $t^{\prime} \approx t+q_{p p k} \mathcal{O}\left(t_{p p k}\right)+q_{s k} \mathcal{O}\left(t_{s k}\right)+q_{p k r} \mathcal{O}\left(t_{p k r}\right)+q_{p k} \mathcal{O}\left(t_{p k}\right)+$ $q_{h_{0}} \mathcal{O}\left(t_{h_{0}}\right)+q_{h_{1}} \mathcal{O}\left(t_{h_{1}}\right)+q_{h_{2}} \mathcal{O}\left(t_{h_{2}}\right)+q_{h_{3}} \mathcal{O}\left(t_{h_{3}}\right)+q_{h_{4}} \mathcal{O}\left(t_{h_{4}}\right)+$ $\mathcal{O}(1),\left(2 q_{h_{1}-\max }\right)$ being the possibly maximal number of queries to $H_{1}, q_{p p k}, q_{s k}, q_{p k r}, q_{p k}, q_{h_{0}}, q_{h_{1}}, q_{h_{2}}, q_{h_{3}}$, and $q_{h_{4}}$ being the numbers of queries to Partial_Private_Key, Secret_Key, Public_Key_Replacement, Public_Key, $\mathrm{H}_{0}, \mathrm{H}_{1}, \mathrm{H}_{2}, \mathrm{H}_{3}$, and $H_{4}$, respectively, and $t_{s i g n}, t_{p p k}, t_{s k}, t_{p k r}, t_{p k}, t_{h_{0}}, t_{h_{1}}, t_{h_{2}}$, $t_{h_{3}}$, and $t_{h_{4}}$ being the computing time of the queries to Sign, Partial_Private_Key, Secret_Key, Public_Key_Replacement, Public_Key, $\mathrm{H}_{0}, \mathrm{H}_{1}, \mathrm{H}_{2}, \mathrm{H}_{3}$, and $\mathrm{H}_{4}$, respectively.

Proof. Assume that a polynomial-time attacker $E_{3}$ wins the game of Definition 8 with probability being at least $\varepsilon$ within running time $t$. A simulator $B$ is given an instance of the $\mathrm{CDH}$ problem $\left\langle G_{1}, q, P, a P, b P\right\rangle$, and $B$ 's goal is to output $a b P$. We will construct $B$ which plays the game in Definition 8 with $E_{3}$ and outputs $a b P$.

Setup. $B$ sets $T_{\text {pub }}=b P$ and chooses $\theta \in\{0,1\}^{*}$ randomly. Then, $B$ publishes $\left\{G_{1}, G_{2}, e, q, P, \theta, T_{\text {pub }}, H_{0}, H_{1}, H_{2}, H_{3}, H_{4}\right\}$. $B$ can respond to the queries from $E_{3}$ as follows.

The simulation for $H_{0}$ is identical to that in the proof of Theorem 13. Consider the following.

(i) $H_{1}$ query: $B$ constructs a list, $H_{1}$-list, and then chooses $\pi \in\left\{1, \ldots, q_{h_{1}-\max }\right\}$ at random and sets an index $j=0$. When $E_{3}$ queries $H_{1}\left(P_{i}\right)$ to $B, B$ checks whether $P_{i}$ is in $H_{1}$-list or not. If $P_{i}$ does not exist in $H_{1}$-list, $B$ computes $j=j+1$ and there are the following two cases. Case 1: if $j=\pi, B$ sets $H_{1}\left(P_{i}\right)=a P$ and stores $\left(P_{i}, a P\right)$ in $H_{1}$-list. Case 2 : if $j \neq \pi, B$ sets $H_{1}\left(P_{i}\right)=\rho_{i} P$ where $\rho_{i}$ is randomly chosen in $Z_{q}^{*}$ and stores $\left(P_{i}, \rho_{i} P, \rho_{i}\right)$ in $H_{1}$-list. Besides, if $P_{i}$ exists in $H_{1}$ list, $B$ gets its mapping value, $\rho_{i} P$ or $a P$. Finally, $B$ returns $\rho_{i} P$ or $a P$.

(ii) $H_{4}$ query: $B$ constructs $H_{4}$-list. If $E_{3}$ queries $H_{4}$ with a string $\varrho$ to $B, B$ checks whether $\varrho$ is in $\mathrm{H}_{4}$-list or not. If $\varrho$ does not exist in $\mathrm{H}_{4}$-list, then there are the following two conditions. Condition 1 : if $\varrho=\theta, B$ sets $W=\beta P=$ $H_{4}(\varrho)$ where $\beta$ is randomly chosen in $Z_{q}^{*}$ and stores $(\varrho, \beta P, \beta)$ in $H_{4}$-list. Condition 2: if $\varrho \neq \theta, B$ sets $W=$ $\Phi=H_{4}(\varrho)$ where $\Phi$ is randomly chosen in $G_{1}$ and stores $(\varrho, \Phi)$ in $\mathrm{H}_{4}$-list. However, if $\varrho$ exists in $\mathrm{H}_{4}$-list, $B$ gets its mapping value, $W=\Phi$ or $\beta P$. Finally, $B$ returns $W$.

(iii) Public_Key query: $B$ constructs a list, $p k$-list. When $E_{3}$ queries Public $\_$Key $\left(I D_{i}\right)$ to $B, B$ looks up $p k$-list.
If $I D_{i}$ is not found in $p k$-list, $B$ does the following works. $B$ computes $P_{i}=x_{i} P$ where $x_{i}$ is randomly chosen in $Z_{q}^{*}$. B queries $H_{1}$ with $P_{i}$. If $H_{1}\left(P_{i}\right)=a P$, $B$ returns "failure"; otherwise, $B$ stores $\left(I D_{i}, P_{i}, x_{i}\right)$ in $p k$-list. Besides, if $I D_{i}$ is found in $p k$-list, $B$ gets $P_{i}$ from $p k$-list. Finally, $B$ returns $P_{i}$ to $E_{3}$.

(iv) Partial_Private_Key query: when $E_{3}$ queries Partial_Private_Key $\left(I D_{i}, P_{i}^{\prime}\right)$ to $B, B$ looks up $H_{0^{-}}$ list, $p k$-list, and $H_{1}$-list. If $I D_{i}$ is not found in $H_{0}$-list, $B$ queries $H_{0}\left(I D_{i}\right)$ and gets $k_{i}$ from $H_{0}$-list; else, $B$ retrieves $k_{i}$ from $H_{0}$-list. If $I D_{i}$ is not found in pk-list, $B$ queries Public_Key with $I D_{i}$ and obtains $P_{i}$ from $p k$-list; otherwise, $B$ catches $P_{i}$ from $p k$-list. If $P_{i} \neq P_{i}^{\prime}, B$ returns "failure." Then, $B$ gets $\rho_{i}$ from $H_{1}$-list. Finally, $B$ returns $\left(D_{i_{0}}=k_{i} b P, D_{i_{1}}=\rho_{i} b P\right)$.

(v) $\mathrm{H}_{2}, \mathrm{H}_{3}$, Secret_Key, and Public_Key_Replacement queries are the same as those in the proof of Theorem 12 . Finally, $E_{3}$ outputs, with probability at least $\varepsilon$, a signature $\sigma^{*}=\left(V^{*}, U_{1}^{*}, U_{2}^{*}, P_{y}^{*}\right)$ on a message $m^{*}$ for the user $I D_{y}$ such that

(1) Verifying $\left(\sigma^{*}, m^{*}, I D_{y}, P_{y}^{*}\right)=$ True;

(2) user $I D_{y}$ has been created;

(3) $P_{y}^{*}$ is different from all of the public keys $P_{y}^{*}$ 's returned by Public Key $\left(I D_{y}\right)$ or used to query Public_Key_Replacement.

From Lemma 9, we fork the sequence of signatures one time and get $\sigma^{\prime}=\left(V^{\prime}, U_{1}^{\prime}, U_{2}^{\prime}, P_{y}^{*}\right)$ on $m^{*}$ by setting $h_{2}^{\prime} \neq h_{2}^{*}$. Thus, we obtain the following two equations:

$$
\begin{gathered}
e\left(P, V^{*}\right)=e\left(T_{\mathrm{pub}}, U_{1}^{*}+h_{2}^{*}\left(Q_{y}+\Gamma_{y}\right)\right) e\left(W, U_{2}^{*}+h_{3}^{*} P_{y}^{*}\right) \\
e\left(P, V^{\prime}\right)=e\left(T_{\mathrm{pub}}, U_{1}^{\prime}+h_{2}^{\prime}\left(Q_{y}+\Gamma_{y}\right)\right) e\left(W, U_{2}^{\prime}+h_{3}^{\prime} P_{y}^{*}\right)
\end{gathered}
$$

with a probability at least $\varepsilon / 2$ by Lemma 10 , where $V^{*} \neq V^{\prime}$, $U_{1}^{*}=U_{1}^{\prime}, h_{2}^{*} \neq h_{2}^{\prime}, U_{2}^{*}=U_{2}^{\prime}$, and $h_{3}^{*}=h_{3}^{\prime}$.

If $H_{1}\left(P_{y}^{*}\right)=a P, B$ can get $a b P=\left(h_{2}^{*}-h_{2}^{\prime}\right)^{-1}\left(V^{*}-V^{\prime}\right)-$ $k_{y} T_{\text {pub }}$ and solve the CDH problem.

The success probability is $\varepsilon^{\prime} \geq \varepsilon /\left(2 q_{h_{1}-\max }\right)$ with the computing time $t^{\prime} \approx t+q_{\text {sign }} \mathcal{O}\left(t_{\text {sign }}\right)+q_{p p k} \mathcal{O}\left(t_{p p k}\right)+q_{s k} \mathcal{O}\left(t_{s k}\right)+$ $q_{p k r} \mathcal{O}\left(t_{p k r}\right)+q_{p k} \mathcal{O}\left(t_{p k}\right)+q_{h_{0}} \mathcal{O}\left(t_{h_{0}}\right)+q_{h_{1}} \mathcal{O}\left(t_{h_{1}}\right)+q_{h_{2}} \mathcal{O}\left(t_{h_{2}}\right)+$ $q_{h_{3}} \mathcal{O}\left(t_{h_{3}}\right)+q_{h_{4}} \mathcal{O}\left(t_{h_{4}}\right)+\mathcal{O}(1)$.

By Theorem 14, a user cannot produce a signature with a new public-secret key pair, which is different from his own one, without the corresponding partial private key being generated from the master secret key. Therefore, if there exist two valid signatures of a user with different public key, the user can prove to anyone that PKG is misbehaving, which means that our scheme achieves Girault's level-3 security.

\section{Discussions}

The comparisons between our scheme and $[5,7,11,13,14,16$, 27-34] are shown in Table 1. Although our proposed scheme 
TABLE 1: The comparisons between $[5,7,11,13,14,16,27-34]$ and our scheme.

\begin{tabular}{|c|c|c|c|c|c|}
\hline & Signing phase & Verification phase & Security level & Formally proved & Security model \\
\hline [5] & $n T_{s}$ & $\begin{array}{l}2 n T_{e}+2 n T_{a} \\
\approx 2400 n t_{m}\end{array}$ & Girault's level-3 & Yes & ROM \\
\hline [7] & $2 n T_{s}+2 n T_{h}+n T_{a}$ & $\begin{array}{c}(2 n+1) T_{p}+n T_{a} \\
+n T_{s}+2 n T_{h} \\
\approx(2475 n+1200) t_{m}\end{array}$ & Girault's level-2 & Yes & ROM \\
\hline$[27]$ & $2 n T_{s}$ & $\begin{array}{l}2 T_{p}+3 n T_{s}+T_{a} \\
\approx(87 n+480) t_{m}\end{array}$ & Girault's level-1 & Yes & ROM \\
\hline [28] Cha-based & $2 n T_{s}$ & $\begin{array}{c}2 T_{p}+2 n T_{s}+n T_{a} \\
+n t_{m} \\
\approx(59 n+480) t_{m}\end{array}$ & Girault's level-1 & No & ROM \\
\hline [28] Waters-based & $(z+2) T_{s}+(z+1) T_{a}$ & $\begin{array}{c}3 T_{p}+2 n(z+1) T_{s} \\
+n(2 z+3) T_{a} \\
\approx(58 n(z+1)+720) t_{m}\end{array}$ & Girault's level-1 & No & STD \\
\hline [28] Hess-based & $n T_{p}+3 n T_{s}+n T_{a}$ & $\begin{array}{c}2 T_{p}+3 n T_{s} \\
+n t_{m} \\
\approx(88 n+480) t_{m}\end{array}$ & Girault's level-1 & No & $\mathrm{ROM}$ \\
\hline [29] Geng-based & $3 n T_{s}+n T_{a}+n t_{m}$ & $\begin{array}{c}3 T_{p}+4 n T_{s} \\
+(n-1) T_{a}+2 n t_{m} \\
\approx(118 n+720) t_{m}\end{array}$ & Girault's level-2 & No & ROM \\
\hline$[32]$ & $2 n T_{p}+11 n T_{s}+4 n\left(T_{a}+t_{m}\right)$ & $\begin{array}{c}2 T_{p}+13 n T_{s} \\
+8 T_{a} \\
\approx(377 n+480) t_{m}\end{array}$ & Girault's level-1 & No & ROM \\
\hline$[11]$ & $2 n T_{s}$ & $\begin{array}{c}(n+1) T_{p} \\
\approx(1200 n+1200) t_{m}\end{array}$ & Girault's level-2 & No & - \\
\hline$[13]$ & $n t_{e}$ & $\begin{aligned} & (n+1) T_{e}+n t_{e} \\
\approx & (1200 n+1440) t_{m}\end{aligned}$ & Girault's level-3 & Yes & ROM \\
\hline$[30]$ & $(k+4) n T_{s}+(k+2) n T_{a}$ & $\begin{array}{c}5 T_{p}+4 n T_{s} \\
+4(n-1) T_{a} \\
\approx(116 n+1200) t_{m}\end{array}$ & Girault's level-1 & No & - \\
\hline [14] & $5 n T_{s}+n T_{a}$ & $\begin{array}{l}(n+1) T_{p}+3 n T_{a}+2 n T_{s} \\
\quad \approx(1258 n+1200) t_{m}\end{array}$ & Girault's level-2 & Yes & ROM \\
\hline [33] & $2 n T_{p}+13 n T_{s}+4 n T_{a}+8 n t_{m}$ & $\begin{aligned} & 2 T_{p}+14 n T_{s} \\
+ & 7 n T_{a}+2 n t_{m} \\
\approx & (408 n+480) t_{m}\end{aligned}$ & Girault's level-1 & No & ROM \\
\hline$[34]$ & $2 n T_{p}+10 n T_{s}+6 n T_{a}+7 n t_{m}$ & $\begin{array}{c}2 T_{p}+13 n T_{s} \\
+n\left(T_{a}+t_{m}\right) \\
\approx(378 n+480) t_{m} \\
\end{array}$ & Girault's level-1 & No & - \\
\hline$[16]$ & $3 n t_{m}+5 n t_{e}$ & $\begin{aligned} & (3 n+1) T_{p}+n t_{e} \\
\approx & (3601 n+1200) t_{m}\end{aligned}$ & Girault's level-2 & Yes & STD \\
\hline$[31]$ & $n\left(T_{s}+T_{a}\right)$ & 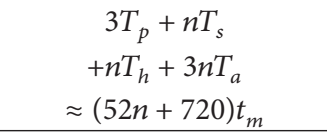 & Girault's level-1 & No & - \\
\hline Ours & $4 n T_{s}+n T_{h}+3 n T_{a}$ & $\begin{array}{c}3 T_{p}+3 n T_{s} \\
+2 n T_{h}+n T_{a} \\
\approx(133 n+720) t_{m}\end{array}$ & Girault's level-3 & Yes & ROM \\
\hline
\end{tabular}

According to [41-43], $T_{p} \approx 5 t_{e}, T_{s} \approx 29 t_{m}, T_{h} \approx 23 t_{m}, T_{a} \approx 0.12 t_{m}$, and $t_{e} \approx 240 t_{m}$.

$z$ : the number of $\ell$-bit chunks in Waters scheme; $k$ : the number of registered users in Qin scheme; $n$ : the number of individual signatures; $T_{p}$ : the time cost of a pairing operation; $T_{s}$ : the time cost of a scalar multiplication in $G_{1} ; T_{h}$ : the time cost of a map-to-point hash operation; $T_{a}$ : the time cost of a point addition operation; $t_{m}$ : the time cost of a modular multiplication in $Z_{p}$; $t_{h}$ : the time cost of a hash operation; ROM: random oracle model; STD: standard model. 
is not the most efficient, it satisfies the property of Girault's level-3 security with formal proofs.

\section{Conclusions}

In this paper, we have proposed a certificateless signature scheme with fast batch verification and it satisfies Girault's level-3 security, where almost all existing signatures for batch verification reach Girault's level-1 security and only one reaches Girault's level-2 security. Finally, we have formally demonstrated that the proposed scheme is unforgeable and achieves Girault's level-3 security based on the CDH problem.

\section{Conflict of Interests}

The authors declare that there is no conflict of interests regarding the publication of this paper.

\section{Acknowledgments}

This work was partially supported by the National Science Council of Taiwan under Grant NSC 102-2219-E-110-002, NSYSU-KMU Joint Research Project (NSYSUKMU 2013I001), and Aim for the Top University Plan of the National Sun Yat-sen University and Ministry of Education, Taiwan. A partial result of this research has been presented at the Eighth Asia Joint Conference on Information Security, Seoul, Republic of Korea, July 25-26, 2013.

\section{References}

[1] A. Shamir, "Identity-based cryptosystems and signature schemes," in Advances in Cryptology-CRYPTO, pp. 47-53, 1985.

[2] B. Libert and J. Quisquater, "What is possible with identity based cryptography for PKIs and what still must be improved," in Proceedings of the European PKI Workshop (EuroPKI'04), pp. 57-70, 2004.

[3] S. S. Al-Riyami and K. G. Paterson, "Certificateless public key cryptography," in Advances in Cryptology-ASIACRYPT, pp. 452473, 2003.

[4] X. Huang, W. Susilo, Y. Mu, and F. Zhang, "On the Security of Certificateless Signature Schemes from Asiacrypt 2003," in Proceedings of the International Conference on Cryptology and Network Security (CANS '05), pp. 13-25, 2005.

[5] Y. Chen, G. Horng, and C. Liu, "Strong non-repudiation based on certificateless short signatures," IET Infomation Security, vol. 7, no. 3, pp. 253-263, 2012.

[6] K. Y. Choi, J. H. Park, and D. H. Lee, "A new provably secure certificateless short signature scheme," Computers and Mathematics with Applications, vol. 61, no. 7, pp. 1760-1768, 2011.

[7] L. Cheng and Q. Wen, "A secure and efficient certificateless short signature scheme," Journal of Engineering Science and Technology Review, vol. 6, no. 2, pp. 35-44, 2011.

[8] H. Du and Q. Wen, "Efficient and provably-secure certificateless short signature scheme from bilinear pairings," Computer Standards and Interfaces, vol. 31, no. 2, pp. 390-394, 2009.

[9] M. Gorantla and A. Saxena, "An efficient certificateless signature scheme," in Proceedings of International Conference on Computational Intelligence and Security (CIS '05), pp. 110-116, 2005.
[10] X. Li, K. Chen, and L. Sun, "Certificateless signature and proxy signature schemes from bilinear pairings," Lithuanian Mathematical Journal, vol. 45, no. 1, pp. 76-83, 2005.

[11] F. Li and P. Liu, "An efficient certificateless signature scheme from bilinear parings," in Proceedings of the International Conference on Network Computing and Information Security (NCIS '11), pp. 35-37, May 2011.

[12] R. Tso, X. Huang, and W. Susilo, "Strongly secure certificateless short signatures," Journal of Systems and Software, vol. 85, no. 6, pp. 1409-1417, 2012.

[13] R. Tso, C. Kim, and X. Yi, "Certificateless message recovery signatures providing Girault's level-3 security," Journal of Shanghai Jiaotong University (Science), vol. 16, no. 5, pp. 577-585, 2011.

[14] Z. Wan, "Certificateless directed signature scheme," in Proceedings of the 7th International Conference on Wireless Communications, Networking and Mobile Computing (WiCOM '11), pp. 1-4, September 2011.

[15] W. Yap, S. Heng, and B. Goi, "An efficient certificateless signature scheme," in Proceedings of International Conference on Emerging Directions in Embedded and Ubiquitous Computing (EUC '06), pp. 322-331, 2006.

[16] Y. Yuan and C. Wang, "A secure certificateless signature scheme in the standard model," Journal of Computational Information Systems, vol. 9, no. 11, pp. 4353-4362, 2013.

[17] Z. Zhang, D. Wong, J. Xu, and D. Feng, "Certificateless publickey signature: security model and efficient construction," in Proceedings of International Conference on Applied Cryptography and Network Securit (ACNS '06), pp. 293-308, 2006.

[18] L. Zhang, Q. Wu, J. Domingo-Ferrer, and B. Qin, "New efficient certificateless signature scheme," in Proceedings of International Conference on Emerging Directions in Embedded and Ubiquitous Computing (EUC '07), pp. 692-703, 2007.

[19] B. C. Hu, D. S. Wong, Z. Zhang, and X. Deng, "Certificateless signature: a new security model and an improved generic construction," Designs, Codes, and Cryptography, vol. 42, no. 2, pp. 109-126, 2007.

[20] M. Girault, "Self-certified public keys," in Advances in Cryptology-EUROCRYPT, pp. 490-497, 1991.

[21] A. Fiat, "Batch RSA," in Advances in cryptology-CRYPTO, pp. 175-185, 1990.

[22] C. Boyd and C. Pavlovski, "Attacking and repairing batch verification schemes," in Advances in cryptology-ASIACRYPT, pp. 58-71, 2000.

[23] L. Harn, "Batch verifying multiple RSA digital signatures," Electronics Letters, vol. 34, no. 12, pp. 1219-1220, 1998.

[24] D. Naccache, D. M'Raihi, S. Vaudenay, and D. Raphaeli, "Can D. S. A. be improved? Complexity trade-offs with the digital signature standard," in Advances in Cryptology-EUROCRYPT, pp. 77-85, 1994.

[25] H. Yoon, J. H. Cheon, and Y. Kim, "Batch verifications with IDbased signatures," in Proceedings of International Conference on Information Security and Cryptology (ICISC '04), pp. 233-248, 2004.

[26] M. Bellare and J. Garay, "Fast batch verification for modular exponentiation and digital signatures," in Advances In Cryptology-EUROCRYPT, pp. 236-250, 1998.

[27] C. Shi, D. Pu, and W. C. Choong, "An efficient identity-based signature scheme with batch verifications," in Proceedings of the 1st International Conference on Scalable information systems (INFOSCALE '06), vol. 22, pp. 1-6, June 2006. 
[28] A. Ferrara, M. Green, S. Hobenberger, and M. Pedersen, "Practical short signature batch verification," in Proceedings of the The Cryptographers' Track at the RSA Conference on Topics in Cryptology (CT-RSA '09), pp. 309-324, 2009.

[29] M. Geng and F. Zhang, "Batch verification for certificateless signature schemes," in Proceedings of the International Conference on Computational Intelligence and Security (CIS '09), pp. 288292, December 2009.

[30] X. Qin, S. Zhang, and L. Jia, "Research on pairing-based batch verification," in Proceedings of the International Conference on Communications and Mobile Computing (CMC '10), pp. 46-50, April 2010.

[31] C. Zhang, P. Ho, and J. Tapolcai, "On batch verification with group testing for vehicular communications," Wireless Networks, vol. 17, no. 8, pp. 1851-1865, 2011.

[32] K. Kim, I. Yie, S. Lim, and D. Nyang, "Batch verification and finding invalid signatures in a group signature scheme," International Journal of Network Security, vol. 13, no. 2, pp. 6170, 2011.

[33] A. Wasef and X. Shen, "Efficient group signature scheme supporting batch verification for securing vehicular networks," in Proceedings of the IEEE International Conference on Communications (ICC '10), pp. 1-5, May 2010.

[34] L. Wei, J. Liu, and T. Zhu, "On a group signature scheme supporting batch verification for vehicular networks," in Proceedings of the 3rd International Conference on Multimedia Information Networking and Security (MINES '11), pp. 436-440, November 2011.

[35] J. Camenisch, S. Hohenberger, and M. Pedersen, "Batch verification of short signature," in Proceedings of the Advances in Cryptology-(EUROCRYPT '07), pp. 246-263, 2007.

[36] T. Cao, D. Lin, and R. Xue, "Security analysis of some batch verifying signatures from pairings," International Journal of Network Security, vol. 3, no. 2, pp. 138-143, 2006.

[37] F. Guo, Y. Mu, and Z. Chen, "Efficient batch verification of short signatures for a single-signer setting without random oracles," in Proceedings of the International Workshop on Security (IWSEC '08), pp. 49-63, 2008.

[38] M. Hwang, C. Lee, and Y. Tang, "Two simple batch verifying multiple digital signatures," in Proceedings of International Conference on Information and Communications Security (ICICS '01), pp. 233-237, 2001.

[39] S. Yen and C. Laih, "Improved digital signature suitable for batch verification," IEEE Transactions on Computers, vol. 44, no. 7, pp. 957-959, 1995.

[40] D. Pointcheval and J. Stern, "Security proofs for signature schemes," in Advances in Cryptology-EUROCRYPT, pp. 387398, 1996.

[41] N. Koblitz, A. Menezes, and S. Vanstone, "The state of elliptic curve cryptography," Designs, Codes, and Cryptography, vol. 19, no. 2-3, pp. 173-193, 2000.

[42] A. Menezes, P. van Oorschot, and S. Vanstone, Handbook of Applied Cryptography, CRC Press, LLC, Boca Raton, Fla, USA, 1997.

[43] Y. Zhang, W. Liu, W. Lou, and Y. Fang, "Securing mobile ad hoc networks with certificateless public keys," IEEE Transactions on Dependable and Secure Computing, vol. 3, no. 4, pp. 386-399, 2006. 


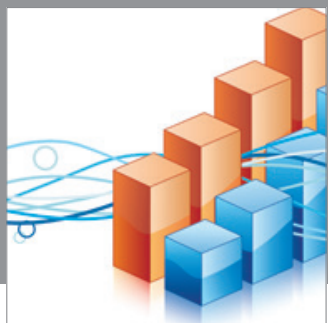

Advances in

Operations Research

mansans

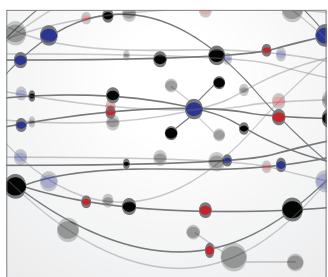

The Scientific World Journal
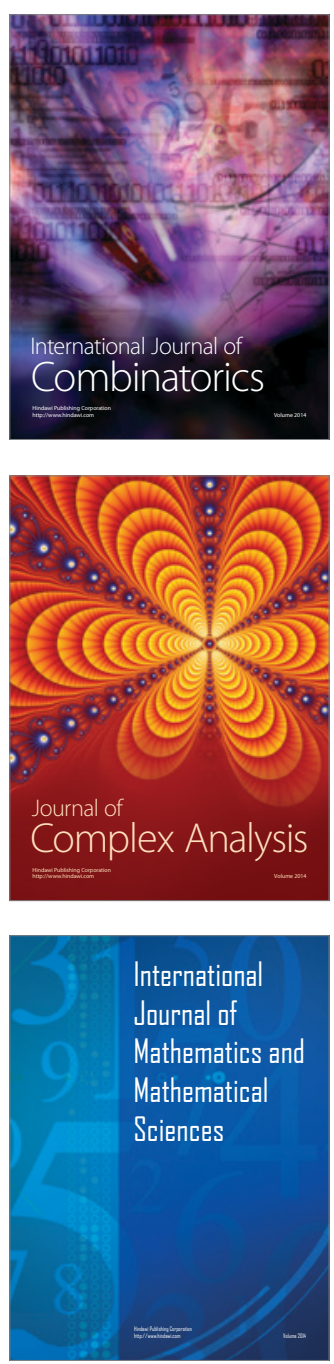
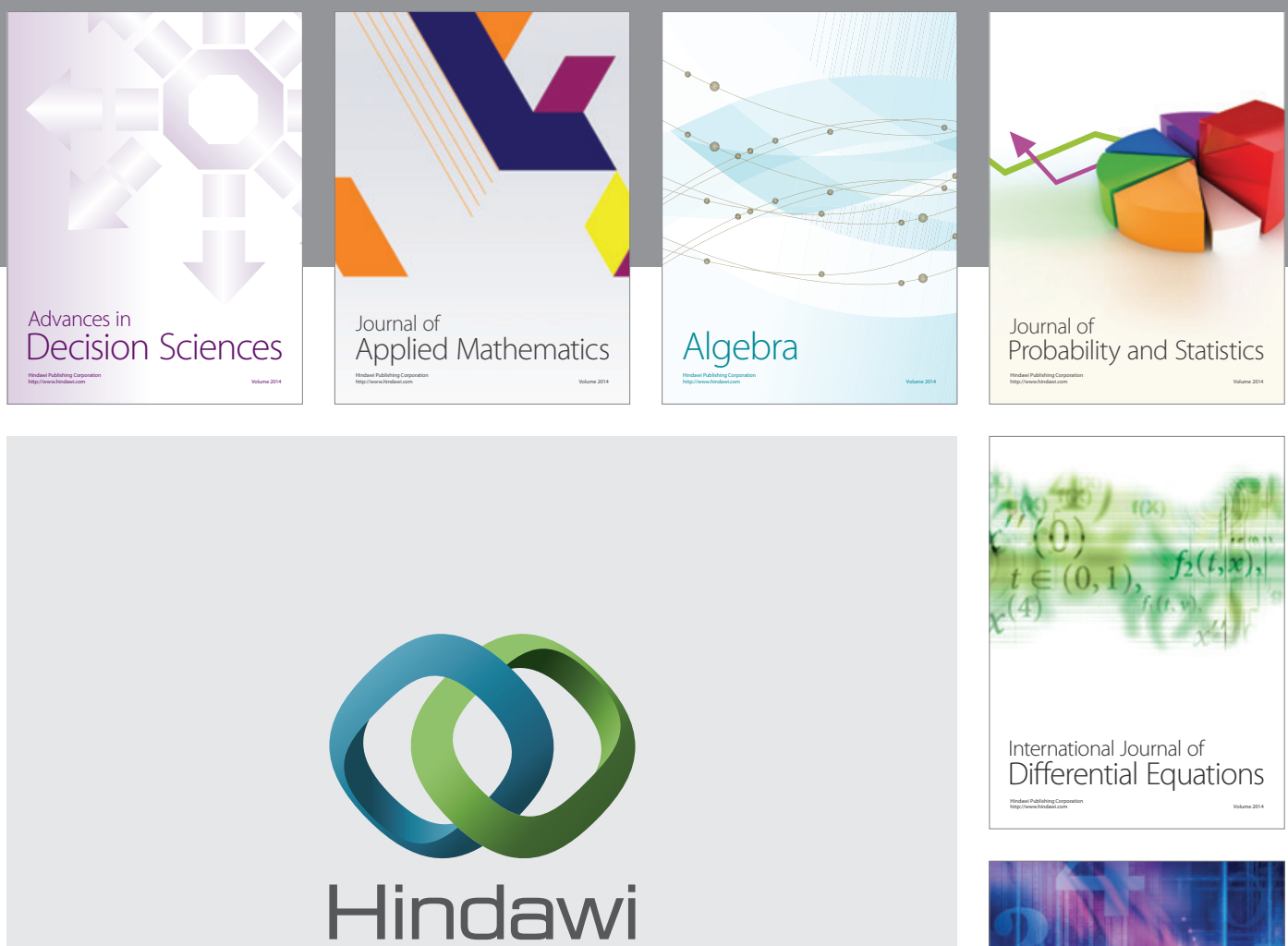

Submit your manuscripts at http://www.hindawi.com
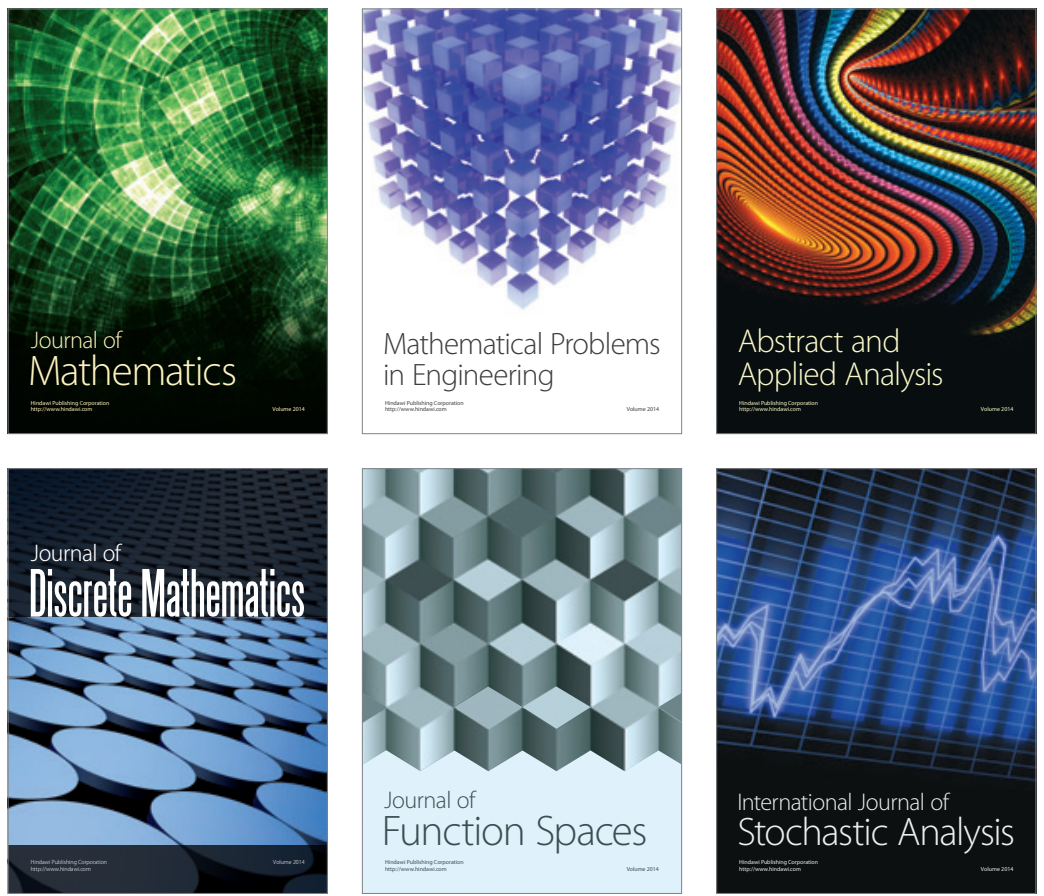

Journal of

Function Spaces

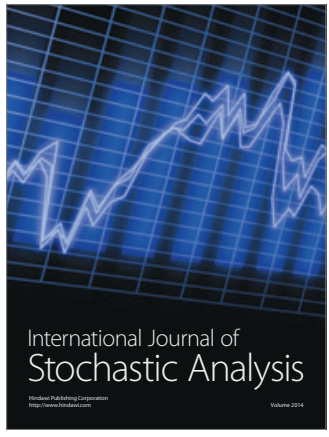

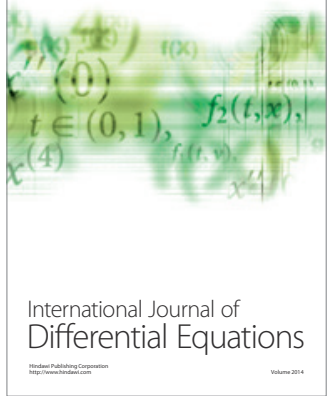
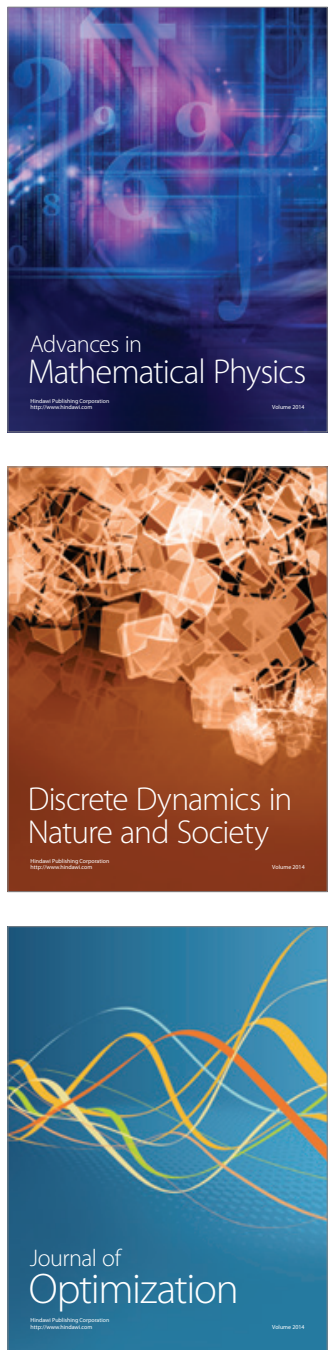